\title{
Discuss the prevention and nursing strategies of clavicular fracture in newborn
}

\author{
Zhang Xiuqing ${ }^{1}$,Hou Lili ${ }^{2}$, Yuan Shengyan ${ }^{1}$, Lu Huichan ${ }^{1}$, Huang Xuanjie $^{2}$, Zhang Jianfang $^{2 \#}$ \\ ${ }^{1}$ Department of Obstetrics and Gynecology, Dongguan People's Hospital. Dong Guang,523000 \\ ${ }^{2}$ Emergency Department, the Seventh Affiliated Hospital, Sun Yat-sen University, Shen Zhen, 518107 \\ \#Zhang Jianfang is the corresponding author. E-mail:edifen@126.com.https://orcid.org/0000-0003-4299-6910
}

Article History Received 5 March 2020 Accepted 25 April 2020 Published 30 June 2020

Cite this Article Zhang Xiuqing, Hou Lili, Yuan Shengyan, Lu Huichan, Huang Xuanjie, Zhang Jianfang. Discuss the prevention and nursing strategies of clavicular fracture in newborn [J].Medical Research, 2020.2(2):1-4, http://dx.doi.org/10.6913/MRHK.202006_2(2).0005

Copyright (C 2020 Creative Publishing Co., Limited. All rights reserved. mrhk26640333@gmail.com.

\section{ABSTRACT}

Objective To summarize the reasons of clavicular fracture in newborn, discuss strategies on the prevention and nursing treatment .

Methods Review and analyze etiopathogenisis of 23 cases with clavicular fracture in newborn from January 2017 to December 2019 in Dongguan People's Hospital (China) and discuss their nursing treatment methods. Results Causes of clavicular fracture in newborn are mostly due to shoulder dystocia and a small part of that result from the birth process anomalies or negligence of medical personnel. All children of clavicle fractures in newborn partially fixed affected limb, can reach clinical cure after six weeks.

Conclusion Correctly assessing the fetal weight, selecting the appropriate mode of parturition, proper handling the birth process anomalies, and establishing a good sense of responsibility with medical personnel can reduce the incidence of clavicular fracture in newborn. Neonatal clavicular fracture after a short partial brake can completely heal without sequela.

Keywords newborn clavicular fracture prevention nursing treatment

\section{INTRODUCTION}

Delivery fractures are fractures that mostly occur during dystocia, often in fetal macrosomia, cephalopelvic disproportion, shoulder dystocia, breech-position delivery and other conditions, which are more common in primipara. Delivery fractures most commonly appear in the clavicle. The causes, preventions and treatments of 23 cases with neonatal clavicle fracture admitted to Dongguan People's Hospital (China) from January 2017 to December 2019 are discussed and summarized as follows.

\section{MATERIALS AND METHODS}

General data: 23 cases with neonatal clavicle fracture were treated during January 2017 to December 2019. General conditions of newborns: Among 23 cases with clavicle fracture, there were 14 males and 9 females, and all full-term infants (gestational age $38 \sim 41$ weeks) with birth weight $2500 \sim 5000 \mathrm{~g}$. The maternal age ranges from 18 to 33 years. There were 19 vaginal deliveries and 4 cesarean sections. Fracture types: 20 cases of transverse fracture, including 8 cases of obvious dislocation, 3 cases of greenstick fracture. Fracture sites: 16 cases of middle-and-outer 
one third segment fractures and 7 cases of middle clavicle fractures.

Diagnosis: Further X-ray examination shall be performed if the newborn has the following situations: a. limited ROM(range of motion) in the upper extremity; b. unexplainable crying after birth; $c$. a hard mass around the clavicle; d. local skin is thicker than that of the healthy side; e. tenderness, bone friction feeling or poor tension and motion in the upper extremity. Clavicle fracture can occasionally be diagnosed through X-ray examinations for amniotic fluid aspiration syndrome or neonatal pneumonia.

Treatment: The family members of the child with greenstick fracture were told to pay attention to conditions of the affected side such as movement, swelling, skin color and so on. Special treatment was not performed. Transverse fracture was treated with Modified Triangular Towel Fixation Method, that is, the affected arm flexed in the sleeve which is buckled by a fibula with a button in front of the upper chest, in order for local immobilization and fixation. Methods: Descriptive analysis was performed on neonates diagnosed with clavicle fracture, and the causes were counted as percentages.

\section{STATISTICAL METHOD}

Statistical analysis was performed by using SPSS17.0 statistical software, and quantitative data was expressed as mean \pm standard deviation $\overline{\mathrm{x}} \pm \mathrm{s}$; comparison between groups was analyzed by variance; $\mathrm{P}<0.05$ indicated that the difference was statistically significant.

\section{RESULT}

To analyze the causes of clavicle fracture in neonates, the neonates with clavicle fracture were divided into vaginal delivery group and cesarean section group (see table 1 and table 2).

\begin{tabular}{lll}
\multicolumn{3}{c}{ Proportion of causes of neonatal clavicle fracture in 19 cases of vaginal delivery group (table 1) } \\
\hline Causes of clavicle fracture & Number of subjects $(\mathrm{n})$ & Percentage $(\%)$ \\
\hline Shoulder dystocia & 8 & 42.11 \\
Fetal macrosomia & 5 & 26.32 \\
Abnormal fetal position & 3 & 15.79 \\
Acute labor & 1 & 5.26 \\
Prolonged second stage & 1 & 5.26 \\
Medical staff misoperation & 1 & 5.26 \\
\hline
\end{tabular}

Proportion of causes of clavicle fracture in 4 neonates in cesarean section group (table 2)

\begin{tabular}{lll}
\hline Causes of clavicle fracture & Number of subjects $(\mathrm{n})$ & Percentage $(\%)$ \\
\hline Medical staff misoperation & 2 & 50.00 \\
Abnormal fetal position & 1 & 25.00 \\
Fetal macrosomia & 1 & 25.00 \\
\hline
\end{tabular}

Prognosis of neonatal clavicle fracture: Among 23 cases with neonatal clavicle fracture, 19 cases were followed up for 6 months, 4 cases were followed up until 6 weeks before losing contact. The follow-up results were as follows: After 2 weeks, the shoulder joint swelling was significantly subsided, and callus formation was observed in the shoulder X-ray film; After 3 weeks, a significant bulge was formed, and the X-ray film suggested a mass of callus formation; After 6 weeks, the child had no limitation of motion, and the shuttle-shaped callus formation was observed. The remodeling shape was ongoing, achieving clinical healing. According to the reexamination after 3 months, the remodeling shape was ongoing, and the bulge was not as obvious as before. After 6 months, the remodeling shape had been almost completed, and the bulge was not palpable.

\section{DISCUSSION}

There are two physiological curves in the clavicle, which are slightly "s" shaped. The inner two-thirds are 
Zhang Xiuqing et al. Discuss the prevention and nursing strategies of clavicular fracture in newborn Medical Research ISSN 2664-0333 eISSN 2664-0341 Volume 2 Issue 2 http://dx.doi.org/10.6913/MRHK.202006_2(2).0005 thick, and the middle and outer one-third is relatively thin, where fractures are likely to occur ${ }^{[1]}$ In older children, clavicle fractures are mostly caused by indirect violence, and sometimes combined with acromioclavicular joint dislocation. However, from the above results, it can be seen that the clavicle fractures are caused by external force during delivery. That reveal they are caused by direct violence, and combination with acromioclavicular joint dislocation is not observed. There are many causes of neonatal clavicle fracture. In vaginal delivery group, shoulder dystocia and fetal macrosomia account for a large proportion. When the fetal weight or shoulder circumference is too large, and it is difficult to get the shoulder out, midwife may increase the traction force and thus cause clavicle fracture. It is particularly obvious with the increase of neonatal weight, and may even lead to more serious birth injury. ${ }^{[2]}$ Neonatal clavicle fracture also tends to take place in abnormal labor, especially in acute delivery. Due to rapid descent of the fetal head, the shoulder quickly squeezed to the pubic symphysis, so that the fragile clavicle quickly bent and fracture occurred. In addition, due to the midwife's lack of experience, when the anterior shoulder of the fetus has not been delivered yet, the posterior shoulder is lifted, resulting in that the clavicle of the anterior shoulder is compressed under the pubic arch and excessive pressure causes the fracture.

However, in the cesarean section group, it can be seen that the primary causes of neonatal clavicle fracture were medical staff's lack of experience or misoperation, of which 2 cases were caused by having not fully estimated the size of the fetus before surgery. The surgical incisions were too small, and excessive force was caused during delivery. Therefore, if the fetal weight can be correctly assessed and appropriate mode of delivery can be selected before delivery of the fetus, the treatment of abnormal labor during delivery can be correctly grasped. A good sense of responsibility of medical staff shall be established at the same time, and the incidence of neonatal clavicle fracture can be significantly reduced. After delivery, the midwives or the surgeons can often tell the abnormality at the first time, and the diagnosis can be confirmed by shoulder X-ray. However, there are still a small number of neonatal clavicle fractures, which could be easy to miss the diagnosis, especially the greenstick fractures do not affect the movement of the newborn, and there are no obvious clinical symptoms and signs. It can only be found accidentally when the newborn undergoes chest X-ray examination due to other diseases such as neonatal amniotic fluid aspiration syndrome and neonatal pneumonia. As a result, thorough examination of each newborn is the key to find the neonatal clavicle fracture. For those with shoulder dystocia and large weight, or in emergent delivery, the clavicle must be listed as a routine examination. Shoulder X-ray examination shall be immediately performed for those with suspicions, which can greatly improve the diagnostic rate of neonatal clavicle fracture. The key to the treatment of neonatal clavicle fracture is to avoid the injury of peripheral blood vessels and nerves at the broken end, so local fixation is particularly important. The treatment methods of clavicle fracture include triangular towel suspension, 8-shaped bandage fixation, open reduction and internal fixation. ${ }^{[3]}$ Clinically, 8-shaped bandage fixation is mostly selected for significantly displaced neonatal clavicle fractures ${ }^{[4]}$. However, because newborns often cry and have a large ROM of the extremities, the fixed bandages often loosen quickly and do not have a fixation effect. Moreover, skin of the newborn is tender. If the 8-shaped bandage is fixed for a long time, most of their skin will be worn and broken. Since 2017, the Modified Triangular Towel Fixation Method has been used in our hospital to treat significantly displaced neonatal clavicle fractures. ${ }^{[5]}$ That is, the affected arm flexed in the sleeve which is buckled by a fibula with a button in front of the upper chest, in order for local immobilization and fixation.

In this way, 23 cases of neonatal clavicle fracture were all cured in 6 weeks. Because of the strong remodeling ability and rapid healing ability of newborns, good molding can generally be completed after 6 weeks. ${ }^{[6]}$ Even if there is severe malalignment, such as severe shortening and angulation deformity, it can also be corrected spontaneously, with few dysfunction of the affected limb in the future. Neonatal clavicle fracture brings pain to children and psychological burden to their families, often causing doctor-patient contradictions. It is necessary to be vigilant and strictly to prevent it. Once it occurs, it is necessary to explain the situation to the children's families, avoid concealing the condition. ${ }^{[7]}$ Parents of the newborn should be comforted by telling 


\section{Zhang Xiuqing et al. Discuss the prevention and nursing strategies of clavicular fracture in newborn Medical Research ISSN 2664-0333 eISSN 2664-0341 Volume 2 Issue 2 http://dx.doi.org/10.6913/MRHK.202006_2(2).0005}

them that due to their excellent fracture healing ability and orthopedic ability, and the prognosis is good. At the same time, excessive treatment is not suggested except for some necessary measurements such as local fixation and pain-relieving.

\section{ACKNOWLEDGEMENT}

This study was supported by Dongguan People's Hospital and the Seventh Affiliated Hospital, Sun Yat-sen University.

The research was performed in Department of Obstetrics and Gynecology, Dongguan People's Hospital.

\section{STATEMENT}

There is no conflict of interest in this article.

\section{REFERENCES}

[1]Rottenstreich M, Rotem R, Bergman M, et al. Isolated neonatal clavicular fracture - is it a risk factor for future shoulder dystocia?[J]. American Journal of Obstetrics and Gynecology, 2020,222S(1):S663-S664.

[2]Rottenstreich M, Rotem R, Bergman M, et al. Isolated neonatal clavicular fracture is a risk factor for future shoulder dystocia[J]. Journal of Maternal-Fetal \& Neonatal Medicine, 2020.

[3]Ahn E S, Jung M S, Lee Y K, et al. Neonatal clavicular fracture: Recent 10 year study[J]. Pediatrics International, 2015,57(1):60-63.

[4]Casellas-Garcia G, Cavanilles-Walker J M, Alberti-Fito G. Clavicular fracture in the newborn: Is fracture location a risk factor for obstetric brachial palsy?[J]. Journal of Neonatal-Perinatal Medicine, 2018,11(1):61-64.

[5]Fadell M, Miller A, Trefan L, et al. Radiological features of healing in newborn clavicular fractures[J]. European Radiology, 2017,27(5):2180-2187.

[6]Zhang Shi Yin. Practical Obstetrics and Gynecology [M]. 2nd Edition. Beijing: People's Medical Publishing House, 2003:539.

[7] evine ME.Birth trauma, incidence and predisposing factors [J]. Obstet Gynecol, 1984.63 (5): 792. 